\title{
Integrative Biological Simulation, Neuropsychology, and AI Safety
}

\author{
Gopal P. Sarma ${ }^{1 *}$, Adam Safron ${ }^{2}$, and Nick J. Hay ${ }^{3 \dagger}$ \\ School of Medicine, Emory University, Atlanta, GA USA \\ Department of Psychology, Northwestern University, Evanston IL USA \\ Vicarious AI, San Francisco, CA USA
}

\begin{abstract}
We describe a biologically-inspired research agenda with parallel tracks aimed at AI and AI safety. The bottomup component consists of building a sequence of biophysically realistic simulations of simple organisms such as the nematode Caenorhabditis elegans, the fruit fly Drosophila melanogaster, and the zebrafish Danio rerio to serve as platforms for research into AI algorithms and system architectures. The top-down component consists of an approach to value alignment that grounds AI goal structures in neuropsychology, broadly considered. Our belief is that parallel pursuit of these tracks will inform the development of value-aligned AI systems that have been inspired by embodied organisms with sensorimotor integration. An important set of side benefits is that the research trajectories we describe here are grounded in long-standing intellectual traditions within existing research communities and funding structures. In addition, these research programs overlap with significant contemporary themes in the biological and psychological sciences such as data/model integration and reproducibility.
\end{abstract}

\section{Introduction}

Bostrom's orthogonality thesis states, under certain weak assumptions, that the intelligence of an agent and its goal structure are independent variables (Bostrom 2014). The orthogonality thesis is a useful conceptual tool for correcting for anthropomorphic bias, i.e. the assumption that an arbitrary agent will behave in a manner similar to human beings. Particularly in a climate of fear and uncertainty about future AI systems, the orthogonality thesis can be a helpful framing to encourage reasoning more clearly about the risks of advanced AI systems and to dislodge concerns arising from science fiction movies and sloppy journalism. However, from an engineering standpoint, it is worth considering that the design of safe, superintelligent AI systems may benefit from examining system architectures in which the intelligent substrate and fundamental goal structure of an agent have been intentionally coupled. We have used the phrase anthropomorphic design to refer to approaches in which AI systems are built to possess commonalities with human neuropsychology (Sarma and Hay 2017,

\footnotetext{
${ }^{*}$ Email: gopal.sarma@emory.edu

${ }^{\dagger}$ The views expressed herein are those of the author and do not necessarily reflect the views of Vicarious AI.
}

Sarma, Hay, and Safron 2018; Sotala 2016).

Anthropomorphic design is best described in reference to the concept of value alignment, which refers to the construction of AI systems that act in accordance with human values throughout their operation (Bostrom 2014, Russell 2016). In recent years, value alignment has been decomposed into specific sub-problems representing possible failure modes of AI systems (Amodei et al. 2016). For instance, consider the sub-problem of "avoiding negative side effects." Often times when we specify a goal, we implicitly assume additional criteria from our model of the world and our value systems which go unstated (Shanahan 2006). As an example, if we have a fully autonomous waste management system to which we give the goal "reduce pollution," we do not want the system to go dump all of the pollution in a neighboring region outside of the range of its sensors, thereby "reducing pollution." A more robust understanding of how to design systems which accomplish their objective while minimizing external impact may allow us to tackle an essential component of value alignment. In a similar vein, "avoiding reward hacking," "scalable oversight," "safe exploration," and "robustness to distributional shift" are all specific properties researchers have identified that should be possessed by value-aligned AI systems (Amodei et al. 2016). Decomposing value alignment into more basic constituents carries the additional benefit that test suites can be designed to verify that agents possess those properties in simulated environments (Leike et al. 2017). Although AI systems today are not powerful enough for value mis-alignment to have particularly negative consequences, the simple frameworks researchers have designed to date are a promising starting point to evolve into substantially more intricate environments for ensuring the safety of future AI systems.

We have previously argued that research in affective neuroscience and related disciplines aimed at grounding human values in neuropsychology may provide important conceptual foundations for understanding value alignment in AI systems. We use neuropsychology in the broadest possible sense of referring to efforts aimed at correlating psychological function with underlying neural architecture. There are several practical benefits that might emerge from 
such a research program, i.e. one aimed at anthropomorphic design. Having more detailed prior information about human values may allow a sophisticated AI system to learn from fewer examples. Similarly, it may enable practical implementations of AI safety techniques that would otherwise be computationally intractable (Sarma and Hay 2017; Sarma, Hay, and Safron 2018).

In this brief position paper, we describe a bottom-up approach to understanding AI architectures that dovetails with both the neuropsychology and test suite based approaches to ensuring value alignment described above. This program is aimed at building realistic biophysical simulations of simple nervous systems which incorporate biomechanics in a simulated environment. Because of key architectural commonalities in the brain plans of vertebrates, and even some invertebrates, we believe that this research is a natural complement to the neuropsychology-based approach to value alignment we have described previously. Our strong intuition is that parallel pursuit of these goals will not only lead to fundamental advances in AI algorithms, but also architectural insights into ensuring value alignment.

The contributions of this manuscript are two-fold: (i) We introduce a set of research objectives in neuroscience that are well positioned to give rise to significant advances in $\mathrm{AI}$ and which have received little attention by the AI safety community. (ii) We suggest two existing approaches to AI safety that may integrate with this research paradigm: a neuropsychology-based approach to value alignment and test suites for agent-based AI systems in simulated environments. We emphasize at the outset that the perspective we take here is largely descriptive. Although we have provided a novel framing, much of the research we discuss here is actively ongoing in a diverse set of communities in the biological sciences. Our objective in this position paper, therefore, is to call attention to a potential path to powerful AI systems which can be entirely justified on their intrinsic value for the biological sciences and which has received little attention by the safety community. It is essential, therefore, as this research progresses at an increasing pace, for parallel steps to be taken to ensure the safety of the resulting systems.

\section{Integrative Biological Simulation}

Claim 1: Simple organisms show complex behavior that continues to be difficult for modern AI systems. Neuronal simulations in virtual environments will allow these biological architectures to be used for AI research.

Integrative biological simulations refer to computational platforms in which diverse, process-specific models, often operating at different scales, are combined into a global, composite model (Sarma and Faundez 2017). Examples include OpenWorm, an internationally coordinated openscience project working towards a realistic biophysical simulation of the nematode Caenorhabditis elegans, Neurokernel, a project with some parallels to OpenWorm aimed at simulating Drosophila melanogaster, Virtual Lamprey, a computational platform for understanding vision and locomotion in the lamprey, BlueBrain, an effort to build a detailed model of the rat cortical micro-column, and the Human Brain Project (HBP), an ambitious successor to BlueBrain which aims to extend this platform to an entire human cerebral cortex (Sarma et al. 2018; Givon and Lazar 2016, Sarvestani et al. 2013, Markram et al. 2015, Amunts et al. 2016). Such platforms may serve as points of integration for data and computational models. The result is a shared structure that can be used by an entire community of researchers to test novel hypotheses, create a tighter feedback loop between experimental and theoretical research, and ensure the reproducibility and robustness of the underlying research output.

In the AI community, awareness of these research programs has primarily been informed by the efforts of BlueBrain and HBP to simulate large regions of mammalian cortical tissue. We are sympathetic in many ways to the aims of these projects. However, we believe that an under-appreciated set of approaches complementing their work consists of using analogous software infrastructure to develop simulations of organisms far below the complexity of mammals or vertebrates. C. elegans, with only 302 neurons, shows simple behavior of learning and memory. Drosophila melanogaster, despite only having $10^{5}$ neurons and no comparable structure to a cerebral cortex, has sophisticated spatial navigation abilities easily rivaling the best autonomous vehicles with a minuscule fraction of the power consumption. The zebrafish Danio rerio has on the order of $10^{7}$ neurons and has been a model system in neuroscience for several decades. Moreover, recent efforts to perform whole-brain functional imaging in the larval zebrafish may make this a particularly attractive target for future integrative simulation platforms (Ahrens et al. 2013). Although much of this research has been motivated by neuroscientific aims and connections to the study of human disease processes, the implications for AI research are significant. Well-engineered software platforms which allow for rapid iteration on existing architectures without the constraints of biological realism will allow AI researchers to test novel hypotheses in embodied organisms in simulated environments [see related work in the animat community (Strannegård et al. 2017; Wilson 1991)]. Real-time visualization of nervous system activity will allow for a deeper understanding of how AI algorithms such as backpropagation, belief propagation, or reinforcement learning may approximate what is observed in nature.

Coupling nervous system activity to drive a simulated body is a tractable approach with organisms such as C. elegans and Drosophila. In OpenWorm, for example, the Boyle-Cohen model of neuromuscular coupling allows for the output of connectome dynamics to drive the activation of body wall muscles and a simulated body (Boyle and Cohen 2008; Gleeson et al. 2018; Palyanov, Khayrulin, and Larson 2018). Similar models are likely achievable with Drosophila as well. Indeed, 
the Neurorobotics Platform of HBP is working towards a general platform for interfacing realistic neural network simulations with robotic bodies (Falotico et al. 2017; Oberts and Sanders 2016). The incorporation of biomechanics into these simulations can be justified on biological grounds. For instance, understanding the effects of anti-psychotic or anti-epileptic medications in model organisms is simplified if researchers can observe changes in behavioral patterns, rather than having to interpret highdimensional data streams of neuronal activity. However, there are reasons to think that sensorimotor integration may be particularly valuable from a purely AI perspective.

As others have argued, despite the significant advances arising from the use of deep representations in neural networks, current AI systems continue to lack many of the qualities of fluid intelligence observed in human beings, particularly in the ability to learn concepts from a relatively small number of examples. One hypothesis is that, unlike modern deep learning systems, human concepts are grounded in rich sensorimotor experience. Despite significant work in transfer learning and domain adaptation, modern systems are largely restricted in their domain of application. The lack of behavior-based concept representation may be a limiting factor in current state-of-the-art systems (Hay et al. 2018, Krichmar 2018, Falotico et al. 2017). Simulations of simple, embodied organisms with realistic virtual environments may provide platforms for AI research aimed at understanding the interplay between concept representation and embodiment. Moreover, used in a modular or hierarchical fashion, contemporary techniques such a deep learning may prove to be powerful components of future iterations of these platforms.

\section{Neuropsychology and Value Alignment Claim 2: Value-alignment research may benefit from insights in neuropsychology and comparative neu- roanatomy.}

We have argued previously for an approach to value alignment which grounds an understanding of human values in neuropsychology (Sarma and Hay 2017; Sarma, Hay, and Safron 2018). In this section, we reproduce the broad outlines of this framework before discussing how these parallel research tracks may come to intersect. Our approach is loosely based on research in affective neuroscience, which aims to categorize emotional universals in the mammalian class and correlate them with an underlying neurological substrate (Panksepp 1998). We use a broad interpretation of the term neuropsychology to denote research aimed at correlating psychological behavior with underlying neural architecture; other related and possibly relevant fields of research include contemplative neuroscience, neuropsychoanalysis, biological anthropology, and comparative neuroanatomy, to name just a few.

It is possible that values and motivations are fundamentally grounded in emotions for human beings. If our emotional substrate is shared with other mammals, or even more broadly with other vertebrates and animals, it suggests that our value systems can be decomposed in ways that inform neuroscience-based AI architectures. For example, one possible (non-exclusive) decomposition of human values is the following:

1. Internal reward systems shared by all mammals: In the taxonomy of affective neuroscience, these include play, panic/grief, fear, rage, seeking, lust, and care. This may also include curiosity and the acquisition of skills.

2. Internal reward systems with human-specific elaborations: For example, uniquely human social behaviors such as family membership, group affiliation, story telling, and gift giving.

3. Products of human deliberation/cognition on our values: The many complex features of value systems produced by several millennia of human social and cultural evolution; likely mediated by cultural inheritance.

An alternate version which we have previously suggested is to view human values as consisting of 1) mammalian values 2) human cognition and 3) several millennia of human social and cultural evolution (Sarma and Hay 2017). Decompositions such as these might allow AI systems to begin with a more nuanced understanding of human values that is then refined over time through observation, hypothesis generation, and human interaction. For an agent that is actively interacting with the world during the learning process, a more informative prior may allow a system to learn from fewer examples, directly translating into a reduced risk of adverse outcomes. Likewise, consider that our values and culture are instilled in children by selective exposure to carefully chosen environments. A neuropsychological understanding of human values may allow us to make similarly strategic choices for AI systems in order to minimize the time required to achieve strong guarantees of value alignment (Evans, Stuhlmüller, and Goodman 2016 Christiano et al. 2017). Moreover, systems with humaninspired architectures may lead to natural avenues for addressing issues of transparency and intelligibility of AI decision making (Wortham, Theodorou, and Bryson 2017 Wachter, Mittelstadt, and Floridi 2006).

\section{Synthesis}

Claim 3: Significant synergy may be achieved by coupling the two research programs described above.

Thus far, we have discussed organisms which lie very far apart on the evolutionary tree. C. elegans and Drosophila possess only $10^{2}-10^{5}$ neurons and the zebrafish Danio rerio roughly $10^{7}$ neurons, whereas mammalian brains range from $10^{8}$ neurons in the brown rat to $10^{10}$ neurons in human neocortex. However, by the time we reach Drosophila we are already confronting a brain with many high-level architectural features which higher animals share, such as two lobes and distinct functional processing regions. Moreover, insects share many neurochemical motivational systems with vertebrates and even higher mammals (Panksepp 1998). Proceeding up the evolutionary 
tree a little further, sophisticated brain centers involved in motor coordination, such as the basal ganglia, are known to be conserved across vertebrates (including zebrafish), and may have homologous structures in arthropods (Grillner and Robertson 2016). In other words, viewed as platforms for research into value-aligned AI systems, there may be clues even from invertebrates and simple vertebrates for how the insights from top-down, neuropsychology-based approaches may be used to design AI systems that possess far greater levels of transparency, intelligibility, and goal structure stability than we see in nature or in our current AI technologies. We believe that a healthy level of interaction between the otherwise disparate communities pursuing these lines of research is the most fruitful way to uncover such clues and to establish clear research directions which lie at the intersection of the two approaches. Moreover, BlueBrain/HBP are already tackling the substantially more difficult challenge of simulating mammalian brains. The success of these projects will only complement insights that arise from approaches oriented towards simulation of simple organisms.

Another point of intersection between integrative biological simulation and current research in AI safety is to extend the concept of test suites for RL agents to the virtual environments of simulated organisms (Leike et al. 2017). As we discussed above, test suites have emerged in the AI safety community as a way to operationalize value alignment into practical, albeit long-term, development strategies for AI systems. By decomposing value alignment into specific subproblems, simulated environments can be created to assess the degree to which artificial agents solve specific tasks while adhering to global safety constraints. Problems such as safe interruptibility, avoiding negative side effects, reward gaming, distributional shift, and others should be adaptable to virtual biological organisms. For example, to what degree do we see variation in susceptibility to reward hacking (i.e. addictive behaviors) in the animal kingdom? Lifting biological constraints, can we augment simulated architectures with modules to reduce the risk of such behavior?

\section{Discussion and Future Directions}

We reiterate that the perspective taken here is largely a descriptive one, as many of the topics we have discussed are actively being pursued by researchers in the biological and psychological sciences. The novel contribution of this manuscript is to frame this research in the context of AI safety. Therefore, in arguing that these two research agendas be "coupled," our intent is to promote community interaction and not necessarily dual-pronged approaches within individual research groups. Given the relative infancy of these ideas, we suspect that much discussion will be necessary before identifying concrete points of overlap.

For the integrative simulation projects, we encourage interested researchers to consult the publications of the respective research groups to find concrete points of entry. For those attracted to expanding the repertoire of simple organisms that have such platforms, there are many commonalities in the necessary software infrastructure, with tools such as NEURON for simulating Hodgkin-Huxley type models, BluePyOpt for extracting kinetic parameters for experimental data, and NetPyNE/Bionet for specifying network models (Hines and Carnevale 1997; Van Geit et al. 2016, Gratiy et al. 2018). Aside from the connectome, an area where there are relevant differences between these organisms is in the gene expression of ion channels. Efforts such as ChannelPedia, NeuroMLDB, ModelDB, and Open Source Brain, all share the goal of enabling storage and re-use of neuroscience data and models (Ranjan et al. 2011; Gleeson et al. 2012). Expanding the scope of these resources to include ion channel data and models for a variety of species would be a key enabler of this research agenda. We suspect that there is literature on comparative neuroanatomy that will give us insights into promising directions to pursue on the lower part of the evolutionary tree.

With regards to the top-down approach to valuealignment, as we emphasized in our previous manuscript, a key obstacle is the widespread concern of reproducibility issues in the biological and psychological literature (Sarma, Hay, and Safron 2018, Sarma 2017). Therefore, we are of the conviction that the most immediate next step is to create a community-driven replication effort aimed at developing a more robust body of knowledge with which to base future research. To that end, we have created a project using the Open Science Framework where we are currently collecting suggestions for candidate studies which would be of high value to either directly replicate or validate through some other means 1 We are particularly interested in using iterated expert elicitation methods such as RAND Corporation's Delphi protocol to encourage consensus building among researchers (Brown 1968).

Finally, regarding the development of test suites for simulated organisms, we have no illusions as to the difficulty of the challenge. Understanding how to translate the highly simplified models of current AI safety frameworks to the complex neural networks of real organisms in realistic physical environments will be a substantial undertaking. However, we believe that such a synthesis is both necessary and desirable, as it may provide insight into hybrid approaches which take advantage of both modern AI and simulated biology to build sophisticated value-aligned systems.

\section{Acknowledgments}

We would like to thank Owain Evans, Tom Everitt, and several anonymous reviewers for insightful discussions and feedback on the manuscript.

\section{References}

[Ahrens et al. 2013] Ahrens, M. B.; Orger, M. B.; Robson, D. N.; et al. 2013. Whole-brain functional imaging at cellu-

1https://tinyurl.com/AI-reproducibility 
lar resolution using light-sheet microscopy. Nature Methods $10(5): 413$.

[Amodei et al. 2016] Amodei, D.; Olah, C.; Steinhardt, J.; Christiano, P.; Schulman, J.; and Mané, D. 2016. Concrete Problems in AI Safety. ArXiv e-prints.

[Amunts et al. 2016] Amunts, K.; Ebell, C.; Muller, J.; et al. 2016. The Human Brain Project: Creating a European Research Infrastructure to Decode the Human Brain. Neuron 92(3):574581.

[Bostrom 2014] Bostrom, N. 2014. Superintelligence: Paths, Dangers, Strategies. Oxford University Press.

[Boyle and Cohen 2008] Boyle, J. H., and Cohen, N. 2008. Caenorhabditis elegans body wall muscles are simple actuators. Biosystems 94(1-2):170-181.

[Brown 1968] Brown, B. B. 1968. Delphi process: A methodology used for the elicitation of opinions of experts. Technical report, Rand Corp Santa Monica CA.

[Christiano et al. 2017] Christiano, P. F.; Leike, J.; Brown, T.; Martic, M.; Legg, S.; and Amodei, D. 2017. Deep reinforcement learning from human preferences. In NIPS, 4299-4307.

[Evans, Stuhlmüller, and Goodman 2016] Evans,

Stuhlmüller, A.; and Goodman, N. D. 2016. Learning the preferences of ignorant, inconsistent agents. In $A A A I$, 323-329.

[Falotico et al. 2017] Falotico, E.; Vannucci, L.; Ambrosano, A.; et al. 2017. Connecting Artificial Brains to Robots in a Comprehensive Simulation Framework: The Neurorobotics Platform. Frontiers in Neurorobotics 11:2.

[Givon and Lazar 2016] Givon, L. E., and Lazar, A. A. 2016. Neurokernel: an open source platform for emulating the fruit fly brain. PLOS ONE 11(1):e0146581.

[Gleeson et al. 2012] Gleeson, P.; Piasini, E.; Crook, S.; et al. 2012. The Open Source Brain Initiative: Enabling Collaborative Modelling in Computational Neuroscience. BMC Neuroscience 13(1): $\mathrm{O} 7$.

[Gleeson et al. 2018] Gleeson, P.; Lung, D.; Grosu, R.; et al. 2018. c302: A multiscale framework for modelling the nervous system of Caenorhabditis elegans. Phil. Trans. R. Soc. B 373(1758):20170379.

[Gratiy et al. 2018] Gratiy, S. L.; Billeh, Y. N.; Dai, K.; et al. 2018. BioNet: A Python interface to NEURON for modeling large-scale networks. PLOS ONE 13(8):e0201630.

[Grillner and Robertson 2016] Grillner, S., and Robertson, B. 2016. The Basal Ganglia over 500 Million Years. Current Biology 26(20):R1088-R1100.

[Hay et al. 2018] Hay, N.; Stark, M.; Schlegel, A.; et al. 2018. Behavior is Everything-Towards Representing Concepts with Sensorimotor Contingencies. In AAAI.

[Hines and Carnevale 1997] Hines, M. L., and Carnevale, N. T. 1997. The NEURON Simulation Environment. Neural Computation 9(6):1179-1209.

[Krichmar 2018] Krichmar, J. L. 2018. Neurorobotics-A Thriving Community and a Promising Pathway Toward Intelligent Cognitive Robots. Front. Neurorobot. 12.

[Leike et al. 2017] Leike, J.; Martic, M.; Krakovna, V.; et al. 2017. AI Safety Gridworlds. arXiv preprint arXiv:1711.09883.
[Markram et al. 2015] Markram, H.; Muller, E.; Ramaswamy, S.; et al. 2015. Reconstruction and Simulation of Neocortical Microcircuitry. Cell 163(2):456-492.

[Oberts and Sanders 2016] Oberts, J., and Sanders, S. 2016. Brain-inspired intelligent robotics: The intersection of robotics and neuroscience. Science/AAAS 1-50.

[Palyanov, Khayrulin, and Larson 2018] Palyanov, A.; Khayrulin, S.; and Larson, S. D. 2018. Three-dimensional simulation of the Caenorhabditis elegans body and muscle cells in liquid and gel environments for behavioural analysis. Phil. Trans. R. Soc. B 373(1758):20170376.

[Panksepp 1998] Panksepp, J. 1998. Affective Neuroscience: The Foundations of Human and Animal Emotions. Oxford University Press.

[Ranjan et al. 2011] Ranjan, R.; Khazen, G.; Gambazzi, L.; et al. 2011. Channelpedia: an integrative and interactive database for ion channels. Front. Neuroinform. 5:36.

[Russell 2016] Russell, S. 2016. Should We Fear Supersmart Robots? Scientific American 314(6):58-59.

[Sarma and Faundez 2017] Sarma, G. P., and Faundez, V. 2017. Integrative biological simulation praxis: Considerations from physics, philosophy, and data/model curation practices. Cellular Logistics 7(4):e1392400.

[Sarma and Hay 2017] Sarma, G. P., and Hay, N. J. 2017. Mammalian Value Systems. Informatica 41(4).

[Sarma et al. 2018] Sarma, G. P.; Lee, C. W.; Portegys, T.; et al. 2018. OpenWorm: Overview and recent advances in integrative biological simulation of Caenorhabditis elegans. Phil. Trans. $R$. Soc. B 373(1758):20170382.

[Sarma, Hay, and Safron 2018] Sarma, G. P.; Hay, N. J.; and Safron, A. 2018. AI Safety and Reproducibility: Establishing Robust Foundations for the Neuropsychology of Human Values. In International Conference on Computer Safety, Reliability, and Security, 507-512. Springer.

[Sarma 2017] Sarma, G. P. 2017. Doing Things Twice (Or Differently): Strategies to Identify Studies for Targeted Validation. arXiv preprint arXiv:1703.01601.

[Sarvestani et al. 2013] Sarvestani, I.; Kozlov, A.; Harischandra, N.; et al. 2013. A computational model of visually guided locomotion in lamprey. Biological Cybernetics 107(5):497-512.

[Shanahan 2006] Shanahan, M. 2006. The Frame Problem. Encyclopedia of Cognitive Science.

[Sotala 2016] Sotala, K. 2016. Defining Human Values for Value Learners. In AAAI Workshop: AI, Ethics, and Society.

[Strannegård et al. 2017] Strannegård, C.; Svangård, N.; Lindström, D.; Bach, J.; and Steunebrink, B. 2017. The Animat Path to Artificial General Intelligence. In Proceedings of IJCAI-17 Workshop on Architectures for Generality \& Autonomy.

[Van Geit et al. 2016] Van Geit, W.; Gevaert, M.; Chindemi, G.; et al. 2016. BluePyOpt: Leveraging Open Source Software and Cloud Infrastructure to Optimise Model Parameters in Neuroscience. Front. Neuroinform. 10:17.

[Wachter, Mittelstadt, and Floridi 2006] Wachter, S.; Mittelstadt, B.; and Floridi, L. 2006. Transparent, Explainable, and Accountable AI for Robotics. Science Robotics 2.

[Wilson 1991] Wilson, S. 1991. The Animat Path to AI. 
[Wortham, Theodorou, and Bryson 2017] Wortham, R. H.; Theodorou, A.; and Bryson, J. J. 2017. Improving robot transparency: real-time visualisation of robot AI substantially improves understanding in naive observers. In 26th IEEE International Symposium on Robot and Human Interactive Communication (RO-MAN), 1424-1431. 